\title{
ON A CLASS OF STOCHASTIC PROCESSES AND ITS RELATIONSHIP TO INFINITE PARTICLE GASES $\left(^{1}\right)$
}

\author{
BY \\ D. PAUL JOHNSON
}

Introduction. Consider the right continuous sample paths $w:[0,+\infty) \rightarrow E$ $=\{+1,-1\}$ with coordinates $x(t)=x_{t}=x_{t}(w) \in E$ together with the $\sigma$-algebras $\mathscr{M}_{t}$ generated by $x(s), s \leqq t$, and assume that for each probability measure $f$ on $E$ and $e \in E$, there exist probability measures $P_{f}$ and $P_{f \mid e}$ on $\mathscr{M}_{\infty}$ for which

(0.A) $P_{f \mid e}(\cdot)=P_{f}(\cdot \mid x(0)=e)$,

(0.B) $P_{f}(x(0)=e)=f(e)$,

(0.C) $P_{f \mid e}\left(x(t+h) \in A \mid \mathscr{M}_{t}\right)=P_{f_{t} \mid x_{t}}(x(h) \in A)$, [a.e. $P_{f \mid e}$ ],

where $A$ is a set of points in $E$ and $f_{t}(A)=P_{f}(x(t) \in A)$. Such a stochastic process will be called a $K$-process. The expression $P_{f \mid e}(\Lambda)$ is to be thought of as the probability that, starting with $x(0)$ distributed according to $f$, the event $\Lambda$ will take place conditional on $x(0)=e$. A $K$-process is a temporally homogeneous Markov process if and only if $P_{f \mid e}$ is independent of $f$, as the reader can easily check. If

$$
\gamma_{e}(u)=\left.\frac{d}{d t} P_{f \mid e}(x(t)=1)\right|_{t=0}, \quad u=f(+1),
$$

then when $\gamma_{+1}$ and $\gamma_{-1}$ are real analytic on the closed interval $[0,1]$, they uniquely determine the distribution of the $K$-process $x(t)$.

In this paper, I shall construct a model of an infinite particle gas with velocities \pm 1 in which the motion of a tagged particle is a $K$-process with specific $\gamma_{ \pm 1}$ and in which the sample paths of any two particles are independent. This will be accomplished by constructing a gas of $n$ like particles, each of which has velocities \pm 1 , and then letting $n \rightarrow \infty$. Each of the $n$ particle gases will be a Markov jump process in which one waits an exponential holding time and then picks an index $i$ according to the uniform distribution $1 / n$ and lets the corresponding particle collide with one or more of the remaining particles. The effect of a collision between a single particle and a set of particles will be a change of state only for the single particle. H. P. McKean [3] has carried out this construction for the case when $\gamma_{ \pm 1}(u)= \pm(u-1)$. In this paper, we generalize his results to the case when $-\gamma_{+1}$

Received by the editors October 12, 1966.

(1) This is essentially a Doctoral Dissertation written under the guidance of Professor Henry P. McKean and submitted to the Department of Mathematics, Massachusetts Institute of Technology, in September 1966. I am especially grateful to Professor McKean for suggesting the problem and for his generous advice.

This research was supported in part by the National Science Foundation (NSF GP 149, NSF GP 2600), and in part by the Office of Naval Research (Nonr 1841 (38)). 
and $\gamma_{-1}$ are both positive on the open interval $(0,1)$ and real analytic on the closed interval $[0,1]$.

The paper is arranged as follows. In $\S 1$ we derive a few basic properties of $K$ processes, including the fact that the functions $\gamma_{+1}$ and $\gamma_{-1}$ uniquely determine the distribution of the process. In $\S 2$, we define the $n$-molecule gases. In $\S 3$, we introduce the basic notions of convergence and equivalence that will be used throughout the paper, and we calculate the limit as $n \rightarrow \infty$ of the generators of the $n$-molecule gases. In $\S 4$ we state the main theorems and finally give their proofs in $\S 5$.

1. $K$-processes. Let $x_{t}, t \in[0, \infty)$ be a $K$-process. Then, defining

$$
P_{f \mid e}(t ; A)=P_{f \mid e}\left(x_{t} \in A\right),
$$

we get a formula for the probabilities of joint observations reminiscent of the case of temporally homogeneous Markov processes.

THEOREM 1. If $x_{t}$ is a K-process, then for $0<t_{1}<\cdots<t_{n}<\infty$,

$$
\begin{aligned}
P_{f \mid e}\left[x\left(t_{1}\right)\right. & \left.\in A_{1}, \ldots, x\left(t_{n}\right) \in A_{n}\right] \\
& =\int_{A_{1}} P_{f \mid e}\left(t_{1} ; d \xi_{1}\right) \int_{A_{2}} P_{f_{t_{1}} \mid \xi_{1}}\left(t_{2}-t_{1} ; d \xi_{2}\right) \cdots \int_{A_{n}} P_{f_{t_{n-1} \mid} \mid \xi_{n-1}}\left(t_{n}-t_{n-1} ; d \xi_{n}\right) .
\end{aligned}
$$

Proof. This is immediate from (0.C).

COROLlary. If $x_{t}$ is a K-process, then

$$
P_{f \mid e}(s+t ; A)=\int P_{f \mid e}(t ; d \xi) P_{f_{t} \mid \xi}(s ; A) .
$$

If $\gamma(u)=u \gamma_{+1}(u)+(1-u) \gamma_{-1}(u)$, then we have the following theorem.

THEOREM 2. If $x_{t}$ is a K-process and if $P_{f \mid e}(t ;+1)$ is differentiable in $t \geqq 0$, then

$$
\begin{gathered}
\frac{d}{d t} f_{t}(+1)=\gamma\left[f_{t}(+1)\right] \\
\frac{d}{d t} P_{f \mid e}(t ;+1)=P_{f \mid e}(t ;+1) \gamma_{+1}\left[f_{t}(+1)\right]+P_{f \mid e}(t ;-1) \gamma_{-1}\left[f_{t}(+1)\right] .
\end{gathered}
$$

Proof. Taking the equation in the Corollary to Theorem 1 and differentiating both sides with respect to $s$ and letting $s=0$, we get (2.B). (2.A) follows from (2.B) if we notice that

$$
f_{t}(+1)=\int f(d e) P_{f \mid e}(t ;+1)
$$

Equation (2.A) of Theorem 2, which is in general nonlinear, has a unique solution bounded by 0 and 1 if $\gamma$ is real analytic in the closed interval $[0,1]$ and if $\gamma(0) \geqq 0$ and $\gamma(1) \leqq 0$. Once the solution of (2.A) is known, (2.B) becomes a linear differential equation for $P_{f \mid e}(t ;+1)$. This equation, in turn, has a unique solution 
bounded by 0 and 1 if $\gamma_{+1}$ and $\gamma_{-1}$ are continuous and $\mp \gamma_{ \pm} \geqq 0$. Having uniquely determined the transition function $P_{f \mid e}$, we can construct the $K$-process by defining probabilities on the cylinder sets in the manner suggested in Theorem 1:

$$
\begin{aligned}
& P\left[x\left(t_{1}\right) \in A_{1}, x\left(t_{2}\right) \in A_{2}, \ldots, x\left(t_{n}\right) \in A_{n}\right] \\
& \quad=\int_{A_{1}} P_{f \mid e}\left(t_{1} ; d \xi_{1}\right) \int_{A_{2}} P_{f_{t_{1}} \mid \xi_{1}}\left(t_{2}-t_{1} ; d \xi_{2}\right) \cdots \int_{A_{n}} P_{f_{t_{n-1}} \mid \xi_{n-1}}\left(t_{n}-t_{n-1} ; d \xi_{n}\right),
\end{aligned}
$$

where $0<t_{1}<\cdots<t_{n}<\infty$. Thus to each pair of functions $-\gamma_{+1}$ and $\gamma_{-1}$ which are positive on the open interval $(0,1)$ and real analytic on the closed interval $[0,1]$, there corresponds a unique $K$-process.

2. $n$-molecule gases. To define the $n$-molecule gases; let $E$ be the set of integers \pm 1 , let $L$ be a fixed positive constant and let $C_{N}^{e}(k), e \in E$ and $k \leqq N$ be a family of nonnegative real numbers with the property that when $C_{N}=\max _{k \leqq N, e= \pm 1} C_{N}^{e}(k)$ one has

$$
\sum_{N=1}^{\infty} N^{P} C_{N} \leqq P ! L^{P}, \quad P \geqq 1
$$

We construct the $n$-molecule gases as follows. Let $X_{n}(t)=\left[x_{1}^{n}(t), \ldots, x_{n}^{n}(t)\right]$ be a Markov jump process on the $n$-dimensional space $E^{n}$ with holding time distribution, in the state $\left[e_{1}, \ldots, e_{n}\right]$, equal to

$$
\exp \left[-t \sum_{N=1}^{n-1} n^{-N} \sum_{i, j_{1}, \ldots, j_{N}}^{(n)} C\left(e_{i} \mid e_{j_{1}}, \ldots, e_{j_{N}}\right)\right]
$$

where $\sum_{j_{0}, j_{1}, \ldots, j_{N}}^{(n)}$ denotes the sum taken over all sequences $\left(j_{0}, \ldots, j_{N}\right), 1 \leqq j_{k} \leqq n$, $j_{k} \neq j_{p}$ when $k \neq p$; and where $C\left(e \mid e_{1}, \ldots, e_{N}\right)=C_{N}$ (number of +1 's in the set $\left.e_{1}, \ldots, e_{N}\right)$. Starting at the state $\left[e_{1}, \ldots, e_{n}\right]$, the probability that the first jump is to the state $\left[e_{1}, \ldots, e_{i-1},-e_{i}, e_{i+1}, \ldots, e_{n}\right]$ will be given by

$$
\frac{\sum_{N=1}^{n-1} n^{-N} \sum_{j_{1}, \ldots, j_{N}}^{(n)} C\left(e_{i} \mid e_{j_{1}}, \ldots, e_{j_{N}}\right)}{\sum_{N=1}^{n-1} n^{-N} \sum_{k, j_{1}, \ldots, j_{N}}^{(n)} C\left(e_{k} \mid e_{j_{1}}, \ldots, e_{j_{N}}\right)} .
$$

The generator of the $n$-molecule gas will therefore be

$$
G_{n} \phi\left(e_{1}, \ldots, e_{n}\right)=\sum_{N=1}^{n-1} n^{-N} \sum_{i, j_{1}, \ldots, j_{N}}^{(n)} C\left(e_{i} \mid e_{j_{1}}, \ldots, e_{j_{N}}\right) \Delta_{i} \phi
$$

where $\Delta_{i} \phi=\phi\left(\ldots,-e_{i}, \ldots\right)-\phi\left(\ldots, e_{i}, \ldots\right)$ or zero, depending on whether $\phi$ depends on the variable $e_{i}$ or not.

To calculate the joint probabilities of $M$ molecules in the gas, notice that if

$$
T_{t} \phi\left(e_{1}, \ldots, e_{n}\right)=E\left[\phi\left(X_{t}^{n}\right) \mid X_{0}^{n}=\left(e_{1}, \ldots, e_{n}\right)\right]
$$


is the semigroup associated with the Markov process $X^{n}$, then

$$
T_{t} \phi\left(e_{1}, \ldots, e_{n}\right)=\exp \left[t G_{n}\right] \phi\left(e_{1}, \ldots, e_{n}\right) .
$$

Thus for any two functions $\phi$ and $\psi$ on $E^{n}$ and $\xi \in E^{n}$, we have

$$
\begin{aligned}
E\left[\phi\left(X_{s}^{n}\right) \psi\left(X_{s+t}^{n}\right) \mid X_{0}^{n}=\xi\right] & =E\left[\phi\left(X_{s}^{n}\right) E\left[\psi\left(X_{s+t}^{n}\right) \mid X_{s}^{n}\right] \mid X_{0}^{n}=\xi\right] \\
& =E\left[\phi\left(X_{s}^{n}\right) T_{t} \psi\left(X_{s}^{n}\right) \mid X_{0}^{n}=\xi\right] \\
& =T_{s}\left(\phi T_{t} \psi\right)(\xi) \\
& =\exp \left[s G_{n}\right] \phi \exp \left[t G_{n}\right] \psi .
\end{aligned}
$$

Using this argument, it is easily shown that if the molecules are initially independent and indentically distributed with distribution $f$ and if $E_{k}=\left(e_{1}^{k}, \ldots, e_{M}^{k}, E, E\right.$, $\ldots, E)$, then the joint distribution of the first $M$ particles is given by

(1)

$$
\begin{aligned}
& P\left[X^{n}\left(t_{1}\right) \in E_{1}, \ldots, X^{n}\left(t_{M}\right) \in E_{M}\right] \\
= & \int f\left(d \xi_{1}\right) \cdots f\left(d \xi_{n}\right) \exp \left[t_{1} G_{n}\right] \chi_{E_{1}} \exp \left[\left(t_{2}-t_{1}\right) G_{n}\right] \chi_{E_{2}} \cdots \exp \left[\left(t_{M}-t_{M-1}\right) G_{n}\right] \chi_{E_{M}},
\end{aligned}
$$

where $\chi_{A}$ is the indicator function of the set $A$.

3. Preliminaries and notation. We wish to calculate the limit of (1) as $n$ goes to infinity. To do this, one might calculate $\lim _{n \rightarrow \infty} G_{n} \phi$ when $\phi$ has a finite number of variables. However, as is evident from (1), this is not necessary as we are only interested in the behavior of $G_{n} \phi$ modulo an integration. Instead, we introduce the following notion of equivalence and convergence.

Definition 3. Let $I$ be the set of all indices $i j$ where $i$ and $j$ are nonnegative integers. Suppose that $\phi$ and $\psi$ are functions whose variables are indexed by indices in $I$ and suppose that $J \subset I$. Then we define

(3.A) If $f$ is a probability measure on $E$, then

$$
\int_{J} f^{\infty} \phi=\int \prod_{\alpha \in J} f\left(d \xi_{\alpha}\right) \phi .
$$

(3.B) $\phi \equiv \psi \bmod J$ if and only if there exist functions $\phi_{j}$ and $\psi_{j}$, whose variables have indices in $I$, and one to one mappings $\delta_{j}$ of $J$ onto $J$ such that

$$
\phi=\sum \phi_{j}, \quad \psi=\sum \psi_{j}
$$

and $\phi_{j}^{\delta}=\psi_{j}$ where $\phi_{j}^{\delta}$ is $\phi_{j}$ with the variables $e_{\delta(\alpha)}$ replacing $e_{\alpha}$ for $\alpha \in J$.

(3.C) $\|\phi\|$ is the sup norm of $\phi$.

(3.D) $\|\phi\|_{J}=\inf _{\psi \equiv \phi \bmod J}\|\psi\|$.

(3.E) $\phi_{n} \rightarrow \phi \bmod J$ as $n \rightarrow \infty$ if and only if $\left\|\phi_{n}-\phi\right\|_{J} \rightarrow 0$ as $n \rightarrow \infty$.

One should note that if $\phi \equiv \psi \bmod J$, then

$$
\int_{J} f^{\infty} \phi=\int_{J} f^{\infty} \psi
$$


Similarly, if $\phi_{n} \rightarrow \phi \bmod J$, then

$$
\int_{J} f^{\infty} \phi_{n} \rightarrow \int_{J} f^{\infty} \phi
$$

It is also easily verified that if $\phi_{1} \equiv \psi_{1} \bmod J, \phi_{2} \equiv \psi_{2} \bmod J$ and $\phi$ has variables whose indices all lie in $J^{c}$, then

and

$$
\phi_{1}+\phi_{2} \equiv \psi_{1}+\psi_{2} \bmod J
$$

$$
\phi \phi_{1} \equiv \phi \psi_{1} \bmod J
$$

These facts will be used throughout the paper without further comment.

Now suppose that $\phi$ is a function of $M$ variables, $M$ finite, which are indexed by an index set $J$ of positive integers. We wish to evaluate $\lim _{n \rightarrow \infty} G_{n} \phi$ modulo $J^{c}$. Clearly we have

$G_{n} \phi\left(e_{1}, \ldots, e_{n}\right)=\sum_{N=1}^{n-1} n^{-N} \sum_{i, j_{1}, \ldots, j_{N}}^{(n)} C\left(e_{i} \mid e_{j_{1}}, \ldots, e_{j_{N}}\right) \Delta_{i} \phi$

$$
\begin{aligned}
=\sum_{N=1}^{n-1} n^{-N} \sum_{i, j_{1}, \ldots, j_{N}: j_{k} \in J c}^{(n)} C\left(e_{i} \mid e_{j_{1}}, \ldots, e_{j_{N}}\right) \Delta_{i} \phi & \\
& +\sum_{N=1}^{n-1} n^{-N} \sum_{i, j_{1}, \ldots, j_{N} ; \text { some } j_{k} \in J}^{(n)} C\left(e_{i} \mid e_{j_{1}}, \ldots, e_{j_{N}}\right) \Delta_{i} \phi .
\end{aligned}
$$

Since we are only interested in evaluating these sums modulo $J^{c}$, we may rename the indices of variables which are not contained in $J$. Using the new indices $1 j$, and adopting the convention that the indices $0 i$ and $i$ are the same, we find that the first sum (2) is equivalent to

$$
\sum_{N=1}^{n-M} n^{-N}(n-M) \cdots(n-M-N+1) \sum_{i} C\left(e_{0 i} \mid e_{11}, \ldots, e_{1 N}\right) \Delta_{0 i} \phi
$$

modulo $J^{c}$ since

$$
\begin{aligned}
& \sum_{i, j_{1}, \ldots, j_{N}: j_{k} \in J c}^{(n)} C\left(e_{i} \mid e_{j_{1}}, \ldots, e_{j_{N}}\right) \Delta_{i} \phi \\
& \equiv(n-M)(n-M-1) \cdots(n-M-N+1) \sum_{i} C\left(e_{0 i} \mid e_{11}, \ldots, e_{1 N}\right) \Delta_{0 i} \phi\left(\bmod J^{c}\right), \\
& \quad \equiv 0 \text { otherwise. } \quad M+N \leqq n,
\end{aligned}
$$

As $n \rightarrow \infty$, (4) converges absolutely since it is clearly bounded by

$$
2 M\|\phi\| \sum_{N=1}^{\infty} C_{N}
$$


The second sum (3) is bounded by

$$
2 M^{2}\|\phi\| n^{-1} \sum_{N=1}^{\infty} N C_{N} \leqq 2 M^{2}\|\phi\| n^{-1} L
$$

and thus, using the dominated convergence theorem, we see that as $n \rightarrow \infty$, $G_{n} \phi\left(e_{1}, \ldots, e_{N}\right)$ converges modulo $J^{c}$ to

$$
\sum_{N=1}^{\infty} \sum_{i} C\left(e_{0 i} \mid e_{11}, \ldots, e_{1 N}\right) \Delta_{0 i} \phi
$$

Now let the pairs $i j$ of nonnegative integers be indices for variables $e_{i j} \in E$ and let $\psi$ be a function of a subset of these variables. We shall call $i$ the order of the index $i j$ and the order of $\psi$ will be defined as the maximum of the orders of its variables. Let $\psi$ be a function of finite order less than or equal to $p$. Then we define

$$
D_{p} \psi=\sum_{N=1}^{\infty} \sum_{i=0}^{p} \sum_{j=1}^{\infty} C\left(e_{i j} \mid e_{p+1,1}, \ldots, e_{p+1, N}\right) \Delta_{i j} \psi
$$

Clearly the operator $D_{p}$ introduces a new set of variables of order $p+1$. Remembering the convention that the indices $0 i$ and $i$ are the same, we have established for any function $\phi$ whose variables are indexed by a finite set of positive integers $J$ that

$$
G_{n} \phi \rightarrow D_{p} \phi \bmod J^{c}
$$

for $p \geqq 0$.

In general, we will write $D \phi$ instead of $D_{p} \phi$ with the understanding that $D$ always adds variables whose indices are of order at least one higher than the order of the function on which it is operating. If $\phi$ has infinite order, then we will always be able to write $\phi=\sum \phi_{k}$ where $\phi_{k}$ has finite order and then let $D \phi=\sum D \phi_{k}$. Finally, we let

$$
\exp [t D] \phi=\sum_{n=0}^{\infty} \frac{t^{n}}{n !} D^{n} \phi
$$

4. Main theorems. Our first theorem is the following:

THEOREM 4. If $\phi_{1}, \ldots, \phi_{p}$ are bounded functions whose variables have indices in a finite set $J$ of $M$ indices, then for $8 L M\left(t_{1}+\cdots+t_{p}\right)<1$ we have

$$
\exp \left[t_{p} G_{n}\right] \phi_{p} \cdots \exp \left[t_{1} G_{n}\right] \phi_{1} \rightarrow \exp \left[t_{p} D\right] \phi_{p} \cdots \exp \left[t_{1} D\right] \phi_{1} \text { modulo } J^{c},
$$

where the expression on the right is well defined and finite.

Thus as $n \rightarrow \infty$, (1) converges to

$$
\int f^{\infty} \exp \left[t_{1} D\right] \chi_{E_{1}} \exp \left[\left(t_{2}-t_{1}\right) D\right] \chi_{E_{2}} \cdots \exp \left[\left(t_{M}-t_{M-1}\right) D\right] \chi_{E_{M}} .
$$

This limiting distribution can be used to define a combined motion of $M$ molecules for which: 
I. the paths of any two molecules are independent for $16 L t<1$, and

II. the distribution of a tagged particle is that of a $K$-process with

$$
\gamma_{ \pm 1}(u)=\mp \sum_{N=1}^{\infty} \sum_{k=0}^{N} C_{N}^{ \pm 1}(k)\left(\begin{array}{l}
N \\
k
\end{array}\right) u^{k}(1-u)^{N-k}
$$

Finally, we are able to show that

III. any $k$-process for which $-\gamma_{+}$and $\gamma_{-}$are positive on the open interval $(0,1)$ and real analytic on the closed interval $[0,1]$ can be constructed as the motion of a tagged particle in an infinite particle gas as described above.

The proof of $\mathrm{I}$ is contained in the following theorem.

THEOREM 5. If $a_{v}, b_{v} \in E, \nu=1, \ldots$, m and if $0<t_{1}<\cdots<t_{m}$ are real and $16 L t_{m}<1$, then for $i \neq j$ and any initial probability measure $f$ on $E$,

$$
\begin{aligned}
\lim _{n \rightarrow \infty} P\left[x_{i}^{n}\left(t_{v}\right)\right. & \left.=a_{v}, x_{j}^{n}\left(t_{v}\right)=b_{v}, 1 \leqq \nu \leqq m\right] \\
& =\lim _{n \rightarrow \infty} P\left[x_{i}^{n}\left(t_{v}\right)=a_{v}, 1 \leqq \nu \leqq m\right] \lim _{n \rightarrow \infty} P\left[x_{j}^{n}\left(t_{v}\right)=b_{v}, 1 \leqq \nu \leqq m\right] .
\end{aligned}
$$

The proof of this and the following theorem is based on the fact that if $\phi$ and $\psi$ have variables whose indices form disjoint sets whose union is $J$, then their product $\phi \psi$, written $\phi \otimes \psi$ when the indices of their variables are disjoint, is such that

$$
D(\phi \otimes \psi) \equiv \phi \otimes D \psi+\psi \otimes D \phi \bmod J^{c}
$$

Letting $m$ be the maximum order of $\phi$ and $\psi$, this equation is easily extended to

(5) $\quad D^{p}(\phi \otimes \psi)=\sum_{k=0}^{p}\left(\begin{array}{l}p \\ k\end{array}\right)\left(D_{p-1+m} \cdots D_{k+m} \phi\right) \otimes\left(D_{k-1+m} \cdots D_{m} \psi\right) \bmod J^{c}$

(see M. Kac [2] for the terminology and another instance of this phenomenon). If we let

$$
\begin{aligned}
& \chi_{a}^{i}\left(\ldots, e_{i}, \ldots\right)=1 \text { if } e_{i}=a \text {, } \\
& =0 \text { otherwise, } \\
& f_{t}(e)=\lim _{n \rightarrow \infty} P\left[x_{n}^{1}(t)=e\right]=\int f^{\infty} \exp [t D] \chi_{e}^{1}
\end{aligned}
$$

and

$$
P_{f \mid a}(t, b)=\lim _{n \rightarrow \infty} P\left[x_{n}^{1}(t)=b \mid x_{n}^{1}(0)=a\right]=\int_{\{1\}^{c}} f^{\infty} \exp [t D] \chi_{b}^{1}(a, \ldots),
$$

then II is proved in the following theorem which establishes the limiting motion of a fixed molecule in the gas as a $K$-process for which

$$
\gamma_{ \pm 1}(u)=\mp \sum_{N=1}^{\infty} \sum_{k=0}^{N} C_{N}^{ \pm 1}(k)\left(\begin{array}{l}
N \\
k
\end{array}\right) u^{k}(1-u)^{N-k} .
$$


THEOREM 6. For $0<t_{1}<\cdots<t_{m}$ with $8 L t_{m}<1$ and $e_{0}, \ldots, e_{m} \in E$, we have

$$
\begin{aligned}
\int_{\{1\}^{\mathrm{c}}} f^{\infty} \exp \left[t_{1} D\right] \chi_{e_{1}}^{1} \cdots & \exp \left[\left(t_{m}-t_{m-1}\right) D\right] \chi_{e_{m}}^{1}\left(e_{0}\right) \\
& =P_{f \mid e_{0}}\left(t_{1}, e_{1}\right) P_{f_{t_{1}} \mid e_{1}}\left(t_{2}-t_{1}, e_{2}\right) \cdots P_{f_{t_{m-1}} \mid e_{m-1}}\left(t_{m}-t_{m-1}, e_{m}\right)
\end{aligned}
$$

and

$$
\gamma_{ \pm 1}(u)=\left.\frac{d}{d t} P_{f \mid \pm 1}(t ; 1)\right|_{t=0}=\mp \sum_{N=1}^{\infty} \sum_{k=0}^{N} C_{N}^{ \pm 1}(k)\left(\begin{array}{l}
N \\
k
\end{array}\right) u^{k}(1-u)^{N-k} \text {. }
$$

Corollary. For $8 L t<1$,

$$
f_{t}(+1)=\int f^{\infty} \exp [t D] \chi_{+1}^{1}
$$

is the solution of the differential equation

$$
(d / d t) f_{t}(+1)=\gamma\left[f_{t}(+1)\right]
$$

where $\gamma(u)=u \gamma_{+1}(u)+(1-u) \gamma_{-1}(u)$.

To prove III, we introduce the following definition.

Definition 7. Let $H$ be the class of functions $\gamma$, mapping $[0,1]$ into the real numbers, for which there exist positive real numbers $C_{N}(k)$ and $L$ such that if $C_{N}=\max _{k \leqq N} C_{N}(k)$,

where

$$
\gamma(u)=\sum_{N=1}^{\infty} B_{N}(u)
$$

and

$$
B_{N}(u)=\sum_{k=0}^{N} C_{N}(k)\left(\begin{array}{l}
N \\
k
\end{array}\right) u^{k}(1-u)^{N-k}
$$

$$
\sum_{N=1}^{\infty} N^{p} C_{N} \leqq p ! L^{p}, \quad p \geqq 1
$$

Thus if $-\gamma_{+1}$ and $\gamma_{-1}$ are both in $H$, we can construct an associated $K$-process for small $t$ by taking the limiting motion of one coordinate in an $n$-dimensional Markov chain as $n \rightarrow \infty$. The class $H$ can be described more simply as follows.

THEOREM 8. $F \in H$ if and only if $F$ is positive on the open interval $(0,1)$ and real analytic on the closed interval $[0,1]$.

The proofs of Theorems 4 through 8 follow.

5. Proofs.

\section{Proof of Theorem 4.}

LEMMA 9. If $\tau_{k}\left(i_{1}, \ldots, i_{p}\right)$ is the number of integers $i_{1}, \ldots, i_{p}$ equal to $k$ and if

$$
A_{p}=\sum_{i_{p}=1}^{p} \sum_{i_{p}}^{p-1} \ldots \sum_{i_{1}=1}^{1} \tau_{p}\left(i_{1}, \ldots, i_{p}\right) ! \cdots \tau_{1}\left(i_{1}, \ldots, i_{p}\right) !
$$

then $A_{p}=1 \cdot 3 \cdot \cdots \cdot(2 p-1) \leqq 2^{p} p !$. 
Proof. Use induction on $p$. The lemma is certainly true for $p=1$. Suppose that it also holds for $p$. Then

$$
\begin{aligned}
A_{p+1}= & \sum_{i_{p+1}=1}^{p+1} \sum_{i_{p}=1}^{p} \ldots \sum_{i_{1}=1}^{1} \tau_{p+1}\left(i_{1}, \ldots, i_{p+1}\right) ! \cdots \tau_{1}\left(i_{1}, \ldots, i_{p+1}\right) ! \\
= & \sum_{i_{p}+1}^{p+1} \sum_{i_{p}=1}^{p} \ldots \sum_{i_{1}=1}^{1} \tau_{p}\left(i_{1}, \ldots, i_{p+1}\right) ! \cdots \tau_{1}\left(i_{1}, \ldots, i_{p+1}\right) ! \\
= & \sum_{i_{p+1}=1}^{p+1} \sum_{i_{p}=1}^{p} \ldots \sum_{i_{1}=1}^{1} \tau_{p}\left(i_{1}, \ldots, i_{p}\right) ! \cdots \tau_{i_{p+1}+1}\left(i_{1}, \ldots, i_{p}\right) ! \\
= & \sum_{i_{p+1}=1}^{p+1} \sum_{i_{p}=1}^{p} \ldots \sum_{i_{1}=1}^{1}\left[1+\tau_{i_{p+1}}\left(i_{1}, \ldots, i_{p}\right)\right] \tau_{p}\left(i_{1}, \ldots, i_{p}\right) ! \cdots \tau_{1}\left(i_{1}, \ldots, i_{p}\right) ! \\
= & (p+1) \sum_{i_{p}=1}^{p} \ldots \sum_{i_{1}=1}^{1} \tau_{p}\left(i_{1}, \ldots, i_{p}\right) ! \ldots \tau_{1}\left(i_{1}, \ldots, i_{p}\right) ! \\
& +\sum_{i_{p}+1}^{p+1} \sum_{i_{p}=1}^{p} \ldots \sum_{i_{1}=1}^{1} \tau_{i_{p+1}}\left(i_{1}, \ldots, i_{p}\right) \tau_{p}\left(i_{1}, \ldots, i_{p}\right) ! \ldots \tau_{1}\left(i_{1}, \ldots, i_{p}\right) ! \\
= & (p+1) A_{p}+\sum_{i_{p}=1}^{p} \ldots \sum_{i_{1}=1}^{1}\left[\sum_{i_{p}+1}^{p+1} \tau_{i_{p+1}}\left(i_{1}, \ldots, i_{p}\right)\right] \tau_{p}\left(i_{1}, \ldots, i_{p}\right) ! \\
= & (p+1) A_{p}+\sum_{i_{p}=1}^{p} \ldots \sum_{i_{1}=1}^{1} p \tau_{p}\left(i_{1}, \ldots, i_{p}\right) ! \ldots \tau_{1}\left(i_{1}, \ldots, i_{p}\right) ! \\
= & \left.(p+1) A_{p}+p A_{p}=(2 p+1) A_{p}=1 \cdot 3 \cdot 5 \cdots, i_{1}, \ldots, i_{p}\right) !
\end{aligned}
$$

and the lemma is proved.

LEMMA 10. If $p, q$ and $M$ are positive integers and $L \geqq 0$, then

$$
\sum_{N_{1}, \ldots, N_{p}}\left(M+N_{1}+\cdots+N_{p}\right)^{q} C_{N_{p}} \cdots C_{N_{1}} \leqq q !(2 L)^{q} \exp [M / L] 2^{p} .
$$

Proof. If $B_{n}^{m}$ equals the number of ways of picking $0 \leqq k_{m} \leqq \cdots \leqq k_{1} \leqq n$, then $B_{n}^{m}=B_{n-1}^{m}+B_{n}^{m-1}$ and hence $B_{n} \leqq 2^{m+n}$. Thus

$$
\begin{aligned}
\sum_{N_{1}, \ldots, N_{p}} & \left(M+N_{1}+\cdots+N_{p}\right)^{q} C_{N_{p}} \cdots C_{N_{1}} \\
\leqq & \sum_{k=0}^{q}\left(\begin{array}{l}
q \\
k
\end{array}\right)\left[\sum_{N_{1}, \ldots, N_{p-1}} C_{N_{p-1}} \cdots C_{N_{1}}\left(M+N_{1}+\cdots+N_{p-1}\right)^{k}\right]\left[\sum_{N_{p}=1}^{\infty} N_{p}^{q-k} C_{N_{p}}\right] \\
\leqq & \sum_{k=0}^{q} \frac{q !}{k !} L^{q-k} \sum_{N_{1}, \ldots, N_{p-1}} C_{N_{p-1}} \cdots C_{N_{1}}\left(M+N_{1}+\cdots+N_{p-1}\right)^{k} \\
\leqq & \sum_{k_{1}=0}^{q} \frac{q !}{k_{1} !} L^{q-k_{1}} \sum_{k_{2}=0}^{k_{1}} \frac{k_{1} !}{k_{2} !} L^{k_{1}-k_{2}} \cdots \sum_{k_{p}=0}^{k_{p-1}} \frac{k_{p-1} !}{k_{p} !} L^{k_{p-1}-k_{p}} M^{k_{p}} \\
= & q ! L^{q} \sum_{k_{1}=0}^{q} \sum_{k_{2}=0}^{k_{1}} \cdots \sum_{k_{p}=0}^{k_{p-1}} \frac{L^{-k_{p}}}{k_{p} !} M^{k_{p}} \\
\leqq & q ! L^{q} \exp [M / L] B_{q-1}^{p-1} \leqq q !(2 L)^{q} \exp [M / L] 2^{p} .
\end{aligned}
$$


LEMMA 11. There exists a sequence $a_{n}$ with $\lim _{n \rightarrow \infty} a_{n}=0$ for which

$$
\sum_{N=n}^{\infty} N^{p} C_{N} \leqq(p+2) ! L^{p+2} a_{n}, \quad p \geqq 0 .
$$

Proof. Let

$$
a_{n}(p)=\frac{\sum_{N=n}^{\infty} N^{p} C_{N}}{(p+2) ! L^{p+2}}
$$

and

$$
a_{n}=\sum_{q=1}^{\infty} a_{n}(q)<\infty
$$

We need only check that $\lim _{n \rightarrow \infty} a_{n}=0$. But this follows from the monotone convergence theorem, since the functions $a_{n}(\cdot)$ are nonnegative monotone decreasing with $\lim _{n \rightarrow \infty} a_{n}(p)=0$.

Let $E_{N}^{j}=\left(e_{i_{1}}, \ldots, e_{i_{N}}\right)$ be an $N$-tuple of variables for which $i_{k} \neq i_{p}$ when $k \neq p$; the $N$-tuples may be different for different $j$. Using this notation, we have

$$
\begin{aligned}
G_{n} \phi_{p} \cdots G_{n} \phi_{1}= & \sum_{N_{p}=1}^{n-1} n^{-N_{p}} \sum^{(n)} C\left(e_{i_{p}} \mid E_{N_{p}}^{p}\right) \Delta_{i_{p}} \phi_{p} \\
& \sum_{N_{p-1}=1}^{n-1} n^{-N_{p-1}} \sum^{(n)} C\left(e_{i_{p-1}} \mid E_{N_{p-1}}^{p-1}\right) \Delta_{i_{p-1}} \cdots \Delta_{i_{1}} \phi_{1} .
\end{aligned}
$$

We can break this sum into two parts; the first part having each choice of $E_{N_{1}}^{1}, \ldots$, $E_{N_{p}}^{p}$ such that no two sets have a variable in common, and the second being bounded by

$$
\begin{aligned}
2^{p}\left\|\phi_{1}\right\| \cdots\left\|\phi_{p}\right\| & \sum_{\max \left(N_{1}, \ldots, N_{p}\right) \leqq n-1} n^{-N_{1}-\cdots-N_{p}} \\
& \cdot M\left(M+N_{1}\right) \cdots\left(M+N_{1}+\cdots+N_{p}\right) W C_{N_{1}} \cdots C_{N_{p}}
\end{aligned}
$$

where $M\left(M+N_{1}\right) \cdots\left(M+N_{1}+\cdots+N_{p}\right)$ is the number of ways of choosing $e_{i_{1}}, \ldots, e_{i_{p}}$ and

$$
\begin{aligned}
W= & N_{1}\left(N_{2}+\cdots+N_{p}\right) n^{N_{1}+\cdots+N_{p}-1}+N_{2}\left(N_{3}+\cdots+N_{p}\right) n^{N_{1}+\cdots+N_{p}-1} \\
& +\cdots+N_{p-1} N_{p} n^{N_{1}+\cdots+N_{p}-1}
\end{aligned}
$$

$N_{k}\left(N_{k+1}+\cdots+N_{p}\right) n^{N_{1}+\cdots+N_{p}-1}$ bounds the number of ways of having one of the paired variables in $E_{k}$ and one in $E_{k+1} \cup \cdots \cup E_{p}$. Clearly we have

$$
|W| \leqq\left(M+N_{1}+\cdots+N_{p}\right)^{2} n^{N_{1}}+\cdots+N_{p}-1 .
$$

Using this bound for $|W|$ in (7) and using Lemma 10, we have (7) bounded by

$$
\begin{aligned}
n^{-1} 2^{p}\left\|\phi_{1}\right\| \cdots\left\|\phi_{p}\right\| & \sum_{N_{1}, \ldots, N_{p}}\left(M+N_{1}+\cdots+N_{p}\right)^{p+2} C_{N_{1}} \cdots C_{N_{p}} \\
& \leqq n^{-1}\left\|\phi_{1}\right\| \cdots\left\|\phi_{p}\right\| \exp [M / L](p+2) !(8 L)^{p+2}
\end{aligned}
$$


In the first sum, the sets of variables $E_{N_{1}}^{1}, \ldots, E_{N_{p}}^{p}$ are such that no two sets have a variable in common. Thus, since we will be integrating over all of the variables whose indices are outside of $J$, we shall rename those variables by letting $E_{N_{i}}^{i}=$ $\left(e_{i 1}, \ldots, e_{i N_{i}}\right)$. Furthermore, we shall rename the variables whose indices are in $J$ as $e_{01}, e_{02}, \ldots, e_{0 M}$. With this in mind, we have modulo $J^{c}$,

$$
G_{n} \phi_{p} \cdots G_{n} \phi_{1}+\text { error }=\sum_{N_{p}=1}^{n-1} \sum_{i_{p}-1, j_{p}-1 \leqq n} C\left(e_{i_{p-1} j_{p-1}} \mid E_{N_{p}}^{p}\right)
$$

$$
\cdot \Delta_{i_{p-1} j_{p-1}} \phi_{p} \sum_{N_{p-1}=1}^{n-1} \ldots \sum_{N_{1}=1}^{n-1} \sum_{i_{0}, j_{0} \leqq n} C\left(e_{i_{0} j_{0}} \mid E_{N_{1}}^{1}\right) \Delta_{i_{0} j_{0}} \phi_{1} \text {. }
$$

If (9) converges as $n \rightarrow \infty$, it clearly converges to $D \phi_{p} \cdots D \phi_{1}$. To see that it does converge, we use Lemma 9 to show that the tail of the series (9) is bounded by

$$
\begin{aligned}
& \sum_{q=1}^{p} \sum_{N_{p}=1}^{\infty} \cdots \sum_{N_{q}=n}^{\infty} \cdots \sum_{N_{1}=1}^{\infty} 2^{p}\left\|\phi_{1}\right\| \cdots\left\|\phi_{p}\right\| M\left(M+N_{1}\right) \cdots\left(M+N_{1}+\cdots+N_{p}\right) C_{N_{1}} \cdots C_{N_{p}} \\
& =\sum_{q=1}^{p}(2 M)^{p} M\left\|\phi_{1}\right\| \cdots\left\|\phi_{p}\right\| \sum_{N_{p}=1}^{\infty} \ldots \sum_{N_{q}=n}^{\infty} \ldots \sum_{N_{1}=1}^{\infty}\left(\sum_{i_{p}=1}^{p} N_{i_{p}}\right)\left(\sum_{i_{p}-1}^{p-1} N_{i_{p}-1}\right) \\
& \cdots\left(\sum_{i_{1}=1}^{1} N_{i_{1}}\right) C_{N_{1}} \cdots C_{N_{p}} \\
& =(2 M)^{p} M\left\|\phi_{1}\right\| \cdots\left\|\phi_{p}\right\| \sum_{q=1}^{p} \sum_{i_{p}=1}^{p} \ldots \sum_{i_{1}=1}^{1} \sum_{N_{p}=1}^{\infty} \ldots \sum_{N_{q}=n}^{\infty} \ldots \sum_{N_{1}=1}^{\infty} N_{i_{1}} \\
& \cdots N_{i_{p}} C_{N_{1}} \cdots C_{N_{p}} \\
& =(2 M)^{p} M\left\|\phi_{1}\right\| \cdots\left\|\phi_{p}\right\| \sum_{q=1}^{p} \sum_{i_{p}=1}^{p} \cdots \sum_{i_{1}=1}^{1}\left(\sum_{N_{p}=1}^{\infty} N_{p}^{\tau_{p}\left(i_{1} \ldots, i_{p}\right)} C_{N_{p}}\right) \\
& \cdots\left(\sum_{N_{q}=n}^{\infty} N_{q^{q}}^{\tau_{q}\left(i_{1}, \ldots, i_{p}\right)} C_{N_{q}}\right) \cdots\left(\sum_{N_{1}=1}^{\infty} N_{1}^{\left.\tau_{1} i_{1}, \ldots, i_{p}\right)} C_{N_{1}}\right) \\
& \leqq(2 M)^{p} M\left\|\phi_{1}\right\| \cdots\left\|\phi_{p}\right\| a_{n} \sum_{q=1}^{p} \sum_{i_{p}=1}^{p} \cdots \sum_{i_{1}=1}^{1} \tau_{p}\left(i_{1}, \ldots, i_{p}\right) ! L^{\tau_{p}\left(i_{1} \ldots, i_{p}\right)} \\
& \left.\left.\cdots\left[\tau_{q}\left(i_{1}, \ldots, i_{p}\right)+2\right] ! L^{\tau_{q}\left(i_{1}\right.}, \ldots, i_{p}\right)+2 \cdots \tau_{1}\left(i_{1}, \ldots, i_{p}\right) ! L^{\tau_{1}\left(i_{1}\right.}, \ldots, i_{p}\right) \\
& \leqq a_{n}(2 M L)^{p} M L^{2}\left\|\phi_{1}\right\| \cdots\left\|\phi_{p}\right\| \sum_{q=1}^{p}(p+2)(p+1) \sum_{i_{p}=1}^{p} \ldots \sum_{i_{1}=1}^{1} \tau_{p}\left(i_{1}, \ldots, i_{p}\right) ! \\
& \leqq a_{n}(4 M L)^{p} M L^{2}\left\|\phi_{1}\right\| \cdots\left\|\phi_{p}\right\| \sum_{q=1}^{p}(p+2) ! \\
& \cdots \tau_{1}\left(i_{1}, \ldots, i_{p}\right) ! \\
& \leqq a_{n}\left\|\phi_{1}\right\| \cdots\left\|\phi_{p}\right\|(p+3) !(4 L M)^{p+3} \text {. }
\end{aligned}
$$


Thus combining the inequalities (8) and (10), we have

$$
\begin{aligned}
\| G_{n} \phi_{p} \cdots G_{n} \phi_{1}-D \phi_{p} & \cdots D \phi_{1} \|_{J^{c}} \\
& \leqq\left(n^{-1}+a_{n}\right)\left\|\phi_{1}\right\| \cdots\left\|\phi_{p}\right\| \exp [M / L](p+3) !(8 L M)^{p+3}
\end{aligned}
$$

Using (10) with $n=1$, we see that

converges since

$$
\exp \left[t_{m} D\right] \phi_{m} \cdots \exp \left[t_{1} D\right] \phi_{1}
$$

$\left\|\exp \left[t_{m} D\right] \phi_{m} \cdots \exp \left[t_{1} D\right] \phi_{1}\right\|$

$$
\begin{aligned}
& =\left\|\sum_{p_{m}=0}^{\infty} \cdots \sum_{p_{1}=0}^{\infty} \frac{t_{1}^{p_{1}} \cdots t_{m}^{p_{m}}}{p_{1} ! \cdots p_{m} !} D^{p_{m}} \phi_{m} \cdots D^{p_{1}} \phi_{1}\right\| \\
& \leqq \sum_{p_{m}=0}^{\infty} \cdots \sum_{p_{1}=0}^{\infty} \frac{t_{1}^{p_{1}} \cdots t_{m}^{p_{m}}}{p_{1} ! \cdots p_{m} !} a_{1}\left\|\phi_{1}\right\| \cdots\left\|\phi_{m}\right\|\left(p_{1}+\cdots+p_{m}+3\right) !(4 L M)^{p_{1}+\cdots+p_{m}+3} \\
& =a_{1}\left\|\phi_{1}\right\| \cdots\left\|\phi_{m}\right\|(4 L M)^{3} \sum_{q=0}^{\infty} \sum_{p_{1}+\cdots+p_{m}=q}\left(4 L M t_{1}\right)^{p_{1}} \cdots\left(4 L M t_{m}\right)^{p_{m}} \frac{(q+3) !}{p_{1} ! \cdots p_{m} !} \\
& =a_{1}\left\|\phi_{1}\right\| \cdots\left\|\phi_{m}\right\|(4 L M)^{3} \sum_{q=0}^{\infty}(q+3)(q+2)(q+1)\left(4 L M t_{1}+\cdots+4 L M t_{m}\right)^{q} \\
& =6 a_{1}\left\|\phi_{1}\right\| \cdots\left\|\phi_{m}\right\|(4 L M)^{3}\left(1-4 L M t_{1}-\cdots-4 L M t_{m}\right)^{-4}
\end{aligned}
$$

for $\left|4 L M t_{1}+\cdots+4 L M t_{m}\right|<1$.

Finally, using (11) we have

$$
\begin{aligned}
& \left\|\exp \left[t_{m} G_{n}\right] \phi_{m} \cdots \exp \left[t_{1} G_{n}\right] \phi_{1}-\exp \left[t_{m} D\right] \phi_{m} \cdots \exp \left[t_{1} D\right] \phi_{1}\right\|_{J^{c}} \\
& \leqq \sum_{p_{1}=0}^{\infty} \cdots \sum_{p_{m}=0}^{\infty} \frac{t_{1}^{p_{1}} \cdots t_{m}^{p_{m}}}{p_{1} ! \cdots p_{m} !}\left\|G_{n}^{p_{m}} \phi_{m} \cdots G_{n}^{p_{1}} \phi_{1}-D^{p_{m}} \phi_{m} \cdots D^{p_{1}} \phi_{1}\right\|_{J^{c}} \\
& \leqq\left(n^{-1}+a_{n}\right)\left\|\phi_{1}\right\| \cdots\left\|\phi_{m}\right\| \exp [M / L] \sum_{p_{1}=0}^{\infty} \cdots \sum_{p_{m}=0}^{\infty} \frac{t_{1}^{p_{1}} \cdots t_{m}^{p_{m}}}{p_{1} ! \cdots p_{m} !} \\
& \cdot\left(p_{1}+\cdots+p_{m}+3\right) !(8 L M)^{p_{1}+\cdots+p_{m}+3} \\
& \leqq\left(n^{-1}+a_{n}\right)\left\|\phi_{1}\right\| \cdots\left\|\phi_{m}\right\| e^{M / L}\left(1-8 L M t_{1}-\cdots-8 L M t_{m}\right)^{-4} \cdot
\end{aligned}
$$

Letting $n \rightarrow \infty$, the theorem is proved.

Proof of Theorem 5. Using equation (5), we have

$$
\begin{aligned}
\lim _{n \rightarrow \infty} P & {\left[x_{i}^{n}\left(t_{v}\right)=a_{v}, x_{j}^{n}\left(t_{v}\right)=b_{v}, 1 \leqq \nu \leqq m\right] } \\
& =\lim _{n \rightarrow \infty} \int f^{n} \exp \left[t_{1} G_{n}\right] \chi_{a_{1}}^{i} \chi_{b_{1}}^{j} \cdots \exp \left[\left(t_{m}-t_{m-1}\right) G_{n}\right] \chi_{a_{m}}^{i} \chi_{b_{m}}^{j} \\
& =\lim _{n \rightarrow \infty} \sum \frac{t_{1}^{p_{1}} \cdots\left(t_{m}-t_{m-1}\right)^{p_{m}}}{p_{1} ! \cdots p_{m} !} \int f^{n} G_{n}^{p_{1}} \chi_{a_{1}}^{i} \chi_{b_{1}}^{j} \cdots G_{n}^{p_{m}} \chi_{a_{m}}^{i} \chi_{b_{m}}^{j}
\end{aligned}
$$




$$
\begin{aligned}
& =\sum \frac{t_{1}^{p_{1}} \cdots\left(t_{m}-t_{m-1}\right)^{p_{m}}}{p_{1} ! \cdots p_{m} !} \int f^{\infty} D^{p_{1}} \chi_{a_{1}}^{i} \chi_{b_{1}}^{j} \cdots D^{p_{m}} \chi_{a_{m}}^{i} \chi_{b_{m}}^{j} \\
& =\sum \frac{t_{1}^{p_{1}} \cdots\left(t_{m}-t_{m-1}\right)^{p_{m}}}{p_{1} ! \cdots p_{m} !} \int f^{\infty} D^{p_{1}} \chi_{a_{1}}^{i} \chi_{b_{1}}^{j} \cdots D^{p_{m-1}} \chi_{a_{m-1}}^{i} \chi_{b_{m-1}}^{j} \\
& \sum_{k_{m}=0}^{p_{m}}\left(\begin{array}{l}
p_{m} \\
k_{m}
\end{array}\right)\left(D_{k_{m}} \cdots D_{1} \chi_{a_{m}}^{i}\right) \otimes\left(D_{p} \cdots D_{k_{m}+1} \chi_{b_{m}}^{j}\right) \\
& =\sum \frac{t_{1}^{p_{1}} \cdots\left(t_{m}-t_{m-1}\right)^{p_{m}}}{p_{1} ! \cdots p_{m} !} \sum_{k_{m}=0}^{p_{m}}\left(\begin{array}{l}
p_{m} \\
k_{m}
\end{array}\right) \int f^{\infty} D^{p_{1}} \chi_{a_{1}}^{i} \chi_{b_{1}}^{j} \\
& \cdots D^{p_{m-1}}\left(\chi_{a_{m-1}}^{i} D^{k_{m}} \chi_{a_{m}}^{i}\right) \otimes\left(\chi_{b_{m-1}}^{j} D^{p_{m}-k_{m}} \chi_{b_{m}}^{j}\right) \\
& =\sum \frac{t_{1}^{p_{1}} \cdots\left(t_{m}-t_{m-1}\right)^{p_{m}}}{p_{1} ! \cdots p_{m} !} \sum_{k_{m}=0}^{p_{m}} \cdots \sum_{k_{1}=0}^{p_{1}}\left(\begin{array}{l}
p_{m} \\
k_{m}
\end{array}\right) \cdots\left(\begin{array}{l}
p_{1} \\
k_{1}
\end{array}\right) \\
& \cdot \int f^{\infty}\left(D^{k_{1}} \chi_{a_{1}}^{i} \cdots D^{k_{m}} \chi_{a_{m}}^{i}\right) \otimes\left(D^{p_{1}-k_{1}} \chi_{b_{1}}^{j} \cdots D^{p_{m}-k_{m}} \chi_{b_{m}}^{j}\right) \\
& =\sum \frac{t_{1}^{p_{1}} \cdots\left(t_{m}-t_{m-1}\right)^{p_{m}}}{k_{1} ! \cdots k_{m} !\left(p_{1}-k_{1}\right) ! \cdots\left(p_{m}-k_{m}\right) !} . \\
& \cdot \int f^{\infty}\left(D^{k_{1}} \chi_{a_{1}}^{i} \cdots D^{k_{m}} \chi_{a_{m}}^{i}\right) \otimes\left(D^{p_{1}-k_{1}} \chi_{b_{1}}^{j} \cdots D^{p_{m}-k_{m}} \chi_{b_{m}}^{j}\right) \\
& =\left(\int f^{\infty} \exp \left[t_{1} D\right] \chi_{a_{1}}^{i} \cdots \exp \left[\left(t_{m}-t_{m-1}\right) D\right] \chi_{a_{m}}^{i}\right) \\
& \cdot\left(\int f^{\infty} \exp \left[t_{1} D\right] \chi_{b_{1}}^{j} \cdots \exp \left[\left(t_{m}-t_{m-1}\right) D\right] \chi_{b_{m}}^{j}\right) \\
& =\lim _{n \rightarrow \infty} P\left[x_{i}^{n}\left(t_{v}\right)=a_{v}, 1 \leqq \nu \leqq m\right] \lim _{n \rightarrow \infty} P\left[x_{j}^{n}\left(t_{v}\right)=b_{v}, 1 \leqq \nu \leqq n\right] .
\end{aligned}
$$

\section{Proof of Theorem 6. Clearly}

$$
\exp [t D] \phi \otimes \psi \equiv\left(\sum \frac{t^{p}}{p !} D_{2 p} D_{2 p-2} \cdots D_{2} \phi\right) \otimes\left(\sum \frac{t^{p}}{p !} D_{2 p-1} D_{2 p-3} \cdots D_{1} \psi\right)
$$

modulo the indices of variables of $\phi$ and $\psi$ and thus $\exp [t D] \phi \otimes \psi$ converges modulo the indices of variables of $\phi$ and $\psi$ whenever $\exp [t D] \phi$ and $\exp [t D] \psi$ converge. Using this fact, we have for $8 L t<1$ and $\chi_{e}^{i}$ defined as in (6),

$$
\begin{aligned}
\int_{\{1\}^{c}} f^{\infty} & \exp [t D] \chi_{\xi_{1}}^{1} \otimes \cdots \otimes \chi_{\xi_{m}}^{m}\left(e_{0}, \ldots\right) \\
& =\sum_{p=0}^{\infty} \frac{t^{p}}{p !} \int_{\{1\}^{c}} f^{\infty} D^{p} \chi_{\xi_{1}}^{1} \otimes \cdots \otimes \chi_{\xi_{m}}^{m}\left(e_{0}, \ldots\right) \\
& =\sum_{p=0}^{\infty} \frac{t^{p}}{p !} \int_{\{1\}^{c}} f^{\infty} \sum_{k=0}^{p}\left(\begin{array}{l}
p \\
k
\end{array}\right)\left[D_{p} \cdots D_{k+1} \chi_{\xi_{1}}^{1}\left(e_{0}, \ldots\right)\right] \otimes D_{k} \cdots D_{1} \chi_{\xi_{2}}^{2} \cdots \chi_{\xi_{m}}^{m}
\end{aligned}
$$




$$
\begin{aligned}
& =\sum_{p=0}^{\infty} \frac{t^{p}}{p !} \sum_{k=0}^{p}\left(\begin{array}{l}
p \\
k
\end{array}\right)\left[\int_{\{1\}^{c}} f^{\infty} D_{p} \cdots D_{k+1} \chi_{\xi_{1}}^{1}\left(e_{0}, \ldots\right)\right] \int f^{\infty} D_{k} \cdots D_{1} \chi_{\xi_{2}}^{2} \cdots \chi_{\xi_{m}}^{m} \\
& =\sum_{p=0}^{\infty} \sum_{k=0}^{p} \frac{t^{k} t^{p-k}}{k !(p-k) !}\left[\int_{\{1\}^{c}} f^{\infty} D^{p-k} \chi_{\xi_{1}}^{1}\left(e_{0}, \ldots\right)\right] \int f^{\infty} D^{k} \chi_{\xi_{2}}^{2} \cdots \chi_{\xi_{m}}^{m} \\
& =\left[\int_{\{1\}^{c}} f^{\infty} \exp [t D] \chi_{\xi_{1}}^{1}\left(e_{0}, \ldots\right)\right] \int f^{\infty} \exp [t D] \chi_{\xi_{2}}^{2} \cdots \chi_{\xi_{m}}^{m} \\
& =\left[\int_{\{1\}^{c}} f^{\infty} \exp [t D] \chi_{\xi_{1}}^{1}\left(e_{0}, \ldots\right)\right] \prod_{k=2}^{m} \int f^{\infty} \exp [t D] \chi_{\xi_{k}}^{k} .
\end{aligned}
$$

Now suppose that $\phi$ is a function on the product space $E^{m}$. Then for $a, b \in E$ and $8 L t<1$ we have, using (12),

$$
\begin{aligned}
& \int_{\{1\}^{c}} f^{\infty} \exp [t D] \chi_{b}^{1} \phi(a, \ldots) \\
& =\sum_{\xi_{1}, \ldots, \xi_{m}}\left[\int_{\{1\}^{c}} f^{\infty} \exp [t D] \chi_{\xi_{1}}^{1} \otimes \cdots \otimes \chi_{\xi_{m}}^{m}(a, \ldots)\right] \\
& \cdot \chi_{b}^{1}\left(\xi_{1}, \ldots, \xi_{m}\right) \phi\left(\xi_{1}, \ldots, \xi_{m}\right) \\
& \text { (13) }=\sum_{\xi_{1}, \ldots, \xi_{m}}\left[\int_{\{1\}^{c}} f^{\infty} \exp [t D] \chi_{\xi_{1}}^{1}(a, \ldots)\right]\left(\prod_{k=2}^{m} \int f^{\infty} \exp [t D] \chi_{\xi_{k}}^{k}\right) \\
& \cdot \chi_{b}^{1}\left(\xi_{1}, \ldots, \xi_{m}\right) \phi\left(\xi_{1}, \ldots, \xi_{m}\right) \\
& =\sum_{\xi_{1}}\left[\int_{\{1\}^{c}} f^{\infty} \exp [t D] \chi_{\xi_{1}}^{\frac{1}{1}}(a, \ldots)\right] \int_{(1\}^{c}} f_{t}^{\infty} \chi_{b}^{1}\left(\xi_{1}, \ldots\right) \phi\left(\xi_{1}, \ldots\right) \\
& =\int_{\{1\}^{c}} f^{\infty} \exp [t D] \chi_{b}^{1}(a, \ldots) \int_{\{1\}^{c}} f_{t}^{\infty} \phi(b, \ldots) .
\end{aligned}
$$

We can now easily prove the theorem if we notice that (13) holds whenever $\phi$ can be written as an absolutely converging series $\sum \phi_{k}$, where each $\phi_{k}$ has a finite number of variables. Since $\phi=\exp \left[t_{2} D\right] \chi_{e_{2}}^{1} \cdots \exp \left[t_{m}-t_{m-1} D\right] \chi_{e_{m}}^{1}$ is such a function, we have

$$
\begin{aligned}
\int_{\{1\}^{c}} f^{\infty} \exp \left[t_{1} D\right] \chi_{e_{1}}^{1} \cdots \exp \left[\left(t_{m}-t_{m-1}\right) D\right] \chi_{e_{m}}^{1} \\
=\left[\int_{\{1\}^{c}} f^{\infty} \exp \left[t_{1} D\right] \chi_{e_{1}}^{1}\left(e_{0}, \ldots\right)\right] \int_{\{1\}^{c}} f_{t_{1}}^{\infty} \exp \left[\left(t_{2}-t_{1}\right) D\right] \chi_{e_{2}}^{1} \\
\cdots \exp \left[\left(t_{m}-t_{m-1}\right) D\right] \chi_{e_{m}}^{1}\left(e_{1}, \ldots\right) \\
=\left[\int_{\{1\}^{c}} f^{\infty} \exp \left[t_{1} D\right] \chi_{e_{1}}^{1}\left(e_{0}, \ldots\right)\right]\left[\int_{\{1\}^{c}} f_{t_{1}}^{\infty} \exp \left[\left(t_{2}-t_{1}\right) D\right] \chi_{e_{2}}^{1}\left(e_{1}, \ldots\right)\right] \\
\cdot \int_{\{1\}^{c}}\left(f_{t_{1}}\right)_{t_{2}-t_{1}}^{\infty} \exp \left[\left(t_{3}-t_{2}\right) D\right] \chi_{e_{3}}^{1} \cdots \exp \left[\left(t_{m}-t_{m-1}\right) D\right] \chi_{e_{m}}^{1}\left(e_{2}, \ldots\right) .
\end{aligned}
$$


Proceeding in this manner and noting that $\left(f_{t}\right)_{s}=f_{t+s}$, we prove the first part of Theorem 6.

Finally,

$$
\begin{aligned}
\gamma_{ \pm 1}(u) & =\left.\frac{d}{d t} P_{f \mid \pm 1}(t ; 1)\right|_{t=0} \\
& =\left.\frac{d}{d t} \int_{\{1\}} f^{\infty} e^{t D} \chi_{+1}^{1}( \pm 1, \ldots)\right|_{t=0} \\
& =\int_{\{1\}^{c}} f^{\infty} D \chi^{1}+1( \pm 1, \ldots) \\
& =\int_{\{1\}^{c}} f^{\infty} \sum_{N=1}^{\infty} C\left( \pm 1 \mid e_{2,1}, \ldots, e_{2, N}\right)\left[\chi_{+1}(\mp 1)-\chi_{+1}( \pm 1)\right] \\
& =\mp \sum_{N=1}^{\infty} \sum_{k=0}^{N} C_{N}^{ \pm 1}(k)\left(\begin{array}{l}
N \\
k
\end{array}\right) u^{k}(1-u)^{N-k},
\end{aligned}
$$

the differentiation being justified by the absolute convergence of the resulting sum. This completes the proof.

Proof of Theorem 8. A necessary condition for a function to be in $H$ is that it be real analytic, as the following lemma demonstrates.

LEMma 12. If $F \in H$, then $F$ has derivatives of all orders and

Proof.

$$
\left|(d / d u)^{p} F(u)\right| \leqq p !(2 L)^{p} .
$$

$$
\begin{aligned}
\left|\left(\frac{d}{d u}\right)^{p} F(u)\right|= & \left|\left(\frac{d}{d u}\right)^{p} \sum_{N=1}^{\infty} \sum_{k=0}^{N} C_{N}(k)\left(\begin{array}{l}
N \\
k
\end{array}\right) u^{k}(1-u)^{N-k}\right| \\
= & \mid \sum_{q=0}^{p}\left(\begin{array}{l}
p \\
q
\end{array}\right) \sum_{N=p-q}^{\infty} \sum_{k=q}^{N-p+q} C_{N}(k)\left(\begin{array}{l}
N \\
k
\end{array}\right) k \cdots(k-q+1) u^{k-q} \\
& \cdot(N-k) \cdots(N-k-p+q+1)(-1)^{p-q}(1-u)^{N-k-p+q} \mid \\
= & \mid \sum_{q=0}^{p}\left(\begin{array}{l}
p \\
q
\end{array}\right) \sum_{N=p-q} \sum_{k=0}^{N-p} C_{N}(k+q)\left(\begin{array}{c}
N \\
k+q
\end{array}\right)(k+q) \cdots(k+1) \\
\leqq & \sum_{q=0}^{p}\left(\begin{array}{l}
p \\
q
\end{array}\right) \sum_{N=p-q}^{\infty} \frac{N !}{(N-p) !} C_{N} \sum_{k=0}^{N-p}\left(\begin{array}{c}
N-p \\
k
\end{array}\right) u^{k}(1-u)^{N-p-k} \\
\leqq & \sum_{q=0}^{p}\left(\begin{array}{l}
p \\
q
\end{array}\right) \sum_{N=p-q}^{\infty} N^{p} C_{N} \leqq \sum_{q=0}^{p}\left(\begin{array}{l}
p \\
q
\end{array}\right) p ! L^{p}=p !(2 L)^{p} .
\end{aligned}
$$

Notice that the term-wise differentiation is justified by the convergence of the resulting sums. 
Lemma 13. If $F, G \in H$, then $F G \in H$.

Proof. Let

$$
\begin{aligned}
& F(u)=\sum_{N=1}^{\infty} \sum_{k=0}^{N} C_{N}(k)\left(\begin{array}{l}
N \\
k
\end{array}\right) u^{k}(1-u)^{N-k} \\
& G(u)=\sum_{N=1}^{\infty} \sum_{k=0}^{N} d_{N}(k)\left(\begin{array}{l}
N \\
k
\end{array}\right) u^{k}(1-u)^{N-k} .
\end{aligned}
$$

Then

$$
F(u) G(u)=\sum_{N=1}^{\infty} \sum_{k=0}^{N} e_{N}(k)\left(\begin{array}{l}
N \\
k
\end{array}\right) u^{k}(1-u)^{N-k}
$$

where

$$
e_{N}(k)=\left(\begin{array}{c}
N \\
k
\end{array}\right)^{-1} \sum_{N_{1}+N_{2}=N} \sum_{k_{1}+k_{2}=k ;} C_{k_{1} \leqq N_{1}: k_{2} \leqq N_{2}} C_{N_{1}}\left(k_{1}\right) d_{N_{2}}\left(k_{2}\right)\left(\begin{array}{c}
N_{1} \\
k_{1}
\end{array}\right)\left(\begin{array}{c}
N_{2} \\
k_{2}
\end{array}\right) .
$$

Letting $e_{N}=\max _{k \leqq n} e_{N}(k)$, we have

$$
\begin{aligned}
\sum_{N=1}^{\infty} N^{p} e_{N} & \leqq \sum_{N=1}^{\infty} \sum_{k=0}^{N} N^{p} e_{N}(k) \\
& =\sum_{N=1}^{\infty} \sum_{k=0}^{N} N^{p}\left(\begin{array}{l}
N \\
k
\end{array}\right)^{-1} \sum_{N_{1}+N_{2}=N} \sum_{k_{1}+k_{2}=k: k_{1} \leqq N_{1}: k_{2} \leqq N_{2}} C_{N_{1}}\left(k_{1}\right) d_{N_{2}}\left(k_{2}\right)\left(\begin{array}{l}
N_{1} \\
k_{1}
\end{array}\right)\left(\begin{array}{c}
N_{2} \\
k_{2}
\end{array}\right) \\
& =\sum_{N_{1}, N_{2}} \sum_{k_{1} \leqq N_{1} ; k_{2} \leqq N_{2}}\left(N_{1}+N_{2}\right)^{p} C_{N_{1}}\left(k_{1}\right) d_{N_{2}}\left(k_{2}\right)\left(\begin{array}{c}
N_{1}+N_{2} \\
k_{1}+k_{2}
\end{array}\right)^{-1}\left(\begin{array}{c}
N_{1} \\
k_{1}
\end{array}\right)\left(\begin{array}{l}
N_{2} \\
k_{2}
\end{array}\right) \\
& \leqq \sum_{N_{1}, N_{2}} \sum_{k_{1} \leqq N_{1} ; k_{2} \leqq N_{2}}\left(N_{1}+N_{2}\right)^{p} C_{N_{1}}\left(k_{1}\right) d_{N_{2}}\left(k_{2}\right) \\
& \leqq \sum_{N_{1}, N_{2}} N_{1} N_{2} \sum_{q=0}^{p}\left(\begin{array}{l}
p \\
q
\end{array}\right) N_{1}^{q} N_{2}^{p-q} C_{N_{1}} d_{N_{2}} \\
& \leqq \sum_{q=0}^{p}\left(\begin{array}{l}
p \\
q
\end{array}\right)\left(\sum N_{1}^{q+1} C_{N_{1}}\right)\left(\sum N_{2}^{p-q+1} d_{N_{2}}\right) \\
& \leqq \sum_{q=0}^{p}\left(\begin{array}{l}
p \\
q
\end{array}\right)(q+1) ! L^{q+1}(p-q+1) ! L^{p-q+1} \leqq(4 L)^{p+2} p !
\end{aligned}
$$

LEMma 14. If $F \in H$, then $\exp (F) \in H$.

Proof. Let

$$
F(u)=\sum_{N=1}^{\infty} \sum_{k=0}^{N} C_{N}(k)\left(\begin{array}{l}
N \\
k
\end{array}\right) u^{k}(1-u)^{N-k}
$$

Then

$$
\exp [F(u)]=\sum_{M=1}^{\infty} \sum_{j=0}^{M}\left(\begin{array}{c}
M \\
j
\end{array}\right) \hat{C}_{M}(j) u^{j}(1-u)^{M-j}
$$


where

$\hat{C}_{M}(j)=\left(\begin{array}{c}M \\ j\end{array}\right)^{-1} \sum_{n=0}^{\infty}(n !)^{-1} \sum_{N_{1}+\cdots+N_{n}=M} \sum_{k_{1}+\cdots+k_{n}=j}\left(\begin{array}{c}N_{1} \\ k_{1}\end{array}\right)$

in this last expression, $k_{1} \leqq N_{1}, \ldots, k_{n} \leqq N_{n}$. Thus

$\cdots\left(\begin{array}{c}N_{n} \\ k_{n}\end{array}\right) C_{N_{1}}\left(k_{1}\right) \cdots C_{N_{n}}\left(k_{n}\right)$

$$
\begin{aligned}
& \sum_{M=1}^{\infty} M^{p} \hat{C}_{M} \leqq \sum_{M=1}^{\infty} \sum_{j=0}^{M} M^{p} \hat{C}_{M}(j) \\
& =\sum_{M=1}^{\infty} \sum_{j=0}^{M} M^{p}\left(\begin{array}{c}
M \\
j
\end{array}\right)^{-1} \sum_{n=0}^{\infty}(n !)^{-1} \sum_{N_{1}+\cdots+N_{n}=M} \sum_{k_{1}+\cdots+k_{n}=j}\left(\begin{array}{l}
N_{1} \\
k_{1}
\end{array}\right) \\
& \cdots\left(\begin{array}{c}
N_{n} \\
k_{n}
\end{array}\right) C_{N_{1}}\left(k_{1}\right) \cdots C_{N_{n}}\left(k_{n}\right) \quad\left(k_{1} \leqq N_{1}, \ldots, k_{n} \leqq N_{n}\right) \\
& =\sum_{N_{1}, \ldots, N_{n} k_{1} \leq N_{1}, \ldots, k_{n} \leq N_{n}} \sum_{n=0}^{\infty}(n !)^{-1}\left(N_{1}+\cdots+N_{n}\right)^{p} \\
& \cdot\left(\begin{array}{c}
N_{1}+\cdots+N_{n} \\
k_{1}+\cdots+k_{n}
\end{array}\right)^{-1}\left(\begin{array}{c}
N_{1} \\
k_{1}
\end{array}\right) \cdots\left(\begin{array}{c}
N_{n} \\
k_{n}
\end{array}\right) C_{N_{1}}\left(k_{1}\right) \cdots C_{N_{n}}\left(k_{n}\right) \\
& \leqq \sum_{N_{1}, \ldots, N_{n} k_{1} \leq N_{1}, \ldots, k_{n} \leq N_{n}} \sum_{n=0}^{\infty}(n !)^{-1}\left(N_{1}+\cdots+N_{n}\right)^{p} C_{N_{1}} \cdots C_{N_{n}} \\
& \leqq \sum_{N_{1}, \ldots, N_{n}} \sum_{n=0}^{\infty}(n !)^{-1} N_{1} \cdots N_{n}\left(N_{1}+\cdots+N_{n}\right)^{p} C_{N_{1}} \cdots C_{N_{n}} \\
& =\sum_{n=0}^{\infty}(n !)^{-1} \sum_{N_{1}, \ldots, N_{n} s_{1}+\cdots+s_{n}=p} \frac{p !}{s_{1} ! \cdots s_{n} !} \\
& \cdot N_{1}^{s_{1}} \cdots N_{n}^{s_{n}} N_{1} \cdots N_{n} C_{N_{1}} \cdots C_{N_{n}} \\
& =\sum_{n=0}^{\infty}(n !)^{-1} \sum_{s_{1}+\cdots+s_{n}=p} p ! L^{p}\left(s_{1}+1\right) \cdots\left(s_{n}+1\right) \\
& =\sum_{n=0}^{\infty}(n !)^{-1} L^{p} 2 n(2 n+1) \cdots(2 n+p-1) \\
& \leqq(2 L)^{p} p ! \sum_{n=0}^{\infty}(n !)^{-1}\left(\begin{array}{c}
n+p-1 \\
p
\end{array}\right) \\
& \leqq p !(2 L)^{p} \sum_{n=0}^{\infty}(n !)^{-1} 2^{n} 2^{p-1} \leqq p !(4 L)^{p} e^{2} .
\end{aligned}
$$

Lemma 15 (HAUSDORFF [1]). If a polynomial $F$ is positive $(>0)$ on the open interval $(0,1)$, then it can be expressed as

where

$$
F(u)=\sum_{m=0}^{N} a_{m} \chi_{N, m}(u), \quad a_{m} \geqq 0
$$

$$
\chi_{N, m}(u)=\left(\begin{array}{l}
N \\
m
\end{array}\right) u^{m}(1-u)^{N-m},
$$

provided that $N$ is sufficiently large. 
LEMMA 16. If $F$ is a complex valued function on a complex disc $|z| \leqq 1+\delta, \delta>0$, real on the real numbers and analytic on the closed disc $|z| \leqq 1$; then for any sufficiently large real constant $C, F(z)+C \in H$.

Proof. Let $F$ be real on the real line and analytic in the closed disc $|z| \leqq 1$. Then there exists a $\delta>0$ such that $F(z)=\sum_{N=0}^{\infty} \alpha_{N} z^{N}$ for $|z| \leqq 1+\delta$ where

$$
\left|\alpha_{N}\right|=\left|F^{(N)}(0)\right| / N ! \leqq(1+\delta)^{-N} A, \quad A \text { a positive constant. }
$$

Now let

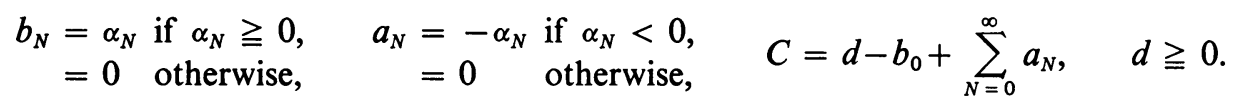

Then

But

$$
F(z)+C=d+\sum_{N=1}^{\infty} b_{N}\left(z^{N}\right)+\sum_{N=1}^{\infty} a_{N}\left(1-z^{N}\right)
$$

and hence, letting

$$
1-z^{N}=\sum_{k=0}^{N-1}\left(\begin{array}{l}
N \\
k
\end{array}\right) z^{k}(1-z)^{N-k}, \quad N \geqq 1,
$$

$$
B_{N}(z)=\sum_{k=0}^{N} C_{N}(k)\left(\begin{array}{l}
N \\
k
\end{array}\right) z^{k}(1-z)^{N-k}
$$

where $C_{1}(0)=a_{1}+d, C_{1}(1)=b_{1}+d$ and

we get

$$
\begin{aligned}
C_{N}(k) & =a_{N} \quad \text { for } 0 \leqq k<N, \quad N>1, \\
& =b_{N} \text { for } k=N,
\end{aligned}
$$

$$
F(z)+C=\sum_{N=1}^{\infty} B_{N}(z) .
$$

Thus we have represented $F+C$ as a sum of Bernstein polynomials when $C \geqq$ $-b_{0}+\sum_{N=0}^{\infty} a_{N}$. We therefore need only show that there exists an $L>0$ such that

But

$$
\sum_{N=1}^{\infty} N^{p} C_{N} \leqq p ! L^{p}, \quad p \geqq 1
$$

Hence

$$
C_{N}=\max _{k \leqq N} C_{N}(k) \leqq(1+\delta)^{-N} A
$$

$$
\sum_{N=0}^{\infty} N^{p} C_{N} \leqq A \sum_{N=0}^{\infty} N^{p} \exp [-N \ln (1+\delta)]
$$

which corresponds to

$$
\int_{0}^{\infty} t^{p} \exp [-t \ln (1+\delta)] d t=\frac{p !}{[\ln (1+\delta)]^{p+1}} \leqq p ! L^{p}
$$

for a suitable $L$.

We can now give sufficient conditions that $F$ be contained in $H$. 
LEMMA 17. If $F$ is analytic on the closed disc $|z| \leqq 1$, real on the reals and positive on $[0,1]$, then $F \in H$.

Proof. Since $F$ is analytic on $|z| \leqq 1$, real on the reals and positive on $[0,1]$, it can be written as $\exp [-C]\left(z-z_{1}\right)\left(z-z_{1}^{*}\right) \cdots\left(z-z_{n}\right)\left(z-z_{n}^{*}\right) \exp [C+G(z)]$ where $z^{*}$ is the conjugate of $z, G$ is analytic on $|z| \leqq 1$ and real on the reals. For $C$ sufficiently large, $C+G(z) \in H$ and hence $\exp [C+G(z)] \in H$. Since, according to Hausdorff's lemma, $\left(z-z_{1}\right)\left(z-z_{1}^{*}\right) \cdots\left(z-z_{n}\right)\left(z-z_{n}^{*}\right) \in H$ and since $H$ is closed under products, the proof is complete.

LEMMA 18. If $F, G \in H$ and $G$ is a polynomial, $0<G(u)<1$ on $(0,1)$, then $F[G(\cdot)] \in H$.

Proof. Let

$$
\begin{aligned}
F(u) & =\sum_{N=1}^{\infty} \sum_{k=0}^{N} C_{N}(k)\left(\begin{array}{l}
N \\
k
\end{array}\right) u^{k}(1-u)^{N-k} \\
G(u) & =\sum_{p=0}^{M} d(p) u^{p}(1-u)^{M-p} \\
1-G(u) & =\sum_{q=0}^{M} e(q) u^{q}(1-u)^{M-q}
\end{aligned}
$$

Then

$$
\begin{aligned}
F[G(u)] & =\sum_{N=1}^{\infty} \sum_{k=0}^{N} C_{N}(k)\left(\begin{array}{c}
N \\
k
\end{array}\right)\left[\sum_{p=0}^{M} d(p) u^{p}(1-u)^{M-p}\right]^{k}\left[\sum_{q=0}^{M} e(q) u^{q}(1-u)^{M-q}\right]^{N-k} \\
& =\sum_{N=1}^{\infty} \sum_{j=0}^{N M} \hat{C}_{N M}(j)\left(\begin{array}{c}
N M \\
j
\end{array}\right) u^{j}(1-u)^{M N-j}
\end{aligned}
$$

where

$$
\begin{aligned}
& \hat{C}_{N M}(j)=\left(\begin{array}{c}
N M \\
j
\end{array}\right)^{-1} \sum_{k=0}^{N} C_{N}(k)\left(\begin{array}{l}
N \\
k
\end{array}\right)_{p_{1}+\cdots+p_{k}+q_{1}+\cdots+q_{N-k}=j ; 0 \leqq p, q \leqq M} \\
& \cdot d\left(p_{1}\right) \cdots d\left(p_{k}\right) e\left(q_{1}\right) \cdots e\left(q_{N-k}\right) .
\end{aligned}
$$

Using Stirling's formula, we have

$$
\begin{aligned}
\left(\begin{array}{c}
N M \\
j
\end{array}\right)^{-1} & \sim(2 \pi M N)^{1 / 2}\left(\frac{j}{M N}\right)^{1 / 2}\left(1-\frac{j}{M N}\right)^{1 / 2}\left(\frac{j}{M N}\right)^{j}\left(1-\frac{j}{M N}\right)^{M N-j} \\
& \leqq 2 \pi M N\left(\frac{j}{M N}\right)^{j}\left(1-\frac{j}{M N}\right)^{M N-j}
\end{aligned}
$$

and thus

$$
\begin{aligned}
& \sum_{N=1}^{\infty}(N M)^{p} \hat{C}_{N M} \leqq \sum_{N=1}^{\infty}(N M)^{p} \sum_{j=0}^{N M} \hat{C}_{N M}(j) \\
&=\sum_{N=1}^{\infty}(N M)^{p} \sum_{j=0}^{N M} \sum_{k=0}^{N} C_{N}(k)\left(\begin{array}{c}
N \\
k
\end{array}\right)\left(\begin{array}{c}
N M \\
j
\end{array}\right)^{-1} \begin{array}{l}
\sum_{p_{1}+\cdots+p_{k}+q_{1}+\cdots+q_{N-k}=j ; 0 \leqq p, q \leqq M} \\
\cdot d\left(p_{1}\right) \cdots d\left(p_{k}\right) e\left(q_{1}\right) \cdots e\left(q_{N-k}\right)
\end{array}
\end{aligned}
$$




$$
\begin{aligned}
& \leqq \sum_{N=1}^{\infty}(N M)^{p} \sum_{j=0}^{N M} \sum_{k=0}^{N} C_{N}(k)\left(\begin{array}{l}
N \\
k
\end{array}\right) 2 \pi M N \sum_{p_{1}+\cdots+p_{k}+q_{1}+\cdots+q_{N-k}=j: 0 \leqq p, q \leqq M} \sum_{\qquad} \cdot d\left(p_{1}\right) \cdots d\left(p_{k}\right) e\left(q_{1}\right) \cdots e\left(q_{N-k}\right)\left(\frac{j}{N M}\right)^{j}\left(1-\frac{j}{N M}\right)^{N M-j} \\
& \leqq 2 \pi M^{p+1} \sum_{N=1}^{\infty} N^{p+1} C_{N} \sum_{m=0}^{M N} \sum_{k=0}^{N}\left(\begin{array}{l}
N \\
k
\end{array}\right) \sum_{j=0}^{N M} \sum_{p_{1}+\cdots+p_{k}+q_{1}+\cdots+q_{N-k}=j ; 0 \leqq p, q \leqq M} \\
& =2 \pi M^{p+1} \sum_{N=1}^{\infty} N^{p+1} C_{N} \sum_{m=0}^{M N} \sum_{k=0}^{N}\left(\begin{array}{l}
N \\
k
\end{array}\right)\left[G\left(\frac{m}{N M}\right)\right]^{k}\left[1-G\left(\frac{m}{N M}\right)\right]^{N-k} \\
& =2 \pi M^{p+2} \sum_{N=1}^{\infty} N^{p+2} C_{N} \\
& \left.\leqq 2 \pi M^{p+2}(p+2) ! p_{k}\right) e\left(q_{1}\right) \cdots e\left(q_{N-k}\right)\left(\frac{m}{N M}\right)^{j+2} \leqq p ! \hat{L}^{p}
\end{aligned}
$$

for suitable $\hat{L}$.

We are now in a position to prove Theorem 8 . If $F \in H$, then $F$ is certainly positive on the open interval $(0,1)$ and, by Lemma 12 , it is real analytic on the closed interval $[0,1]$. Therefore assume that $F$ is positive and real analytic on the closed interval $[0,1]$; if $F$ had roots at 0 or 1 , we could divide through by them. Then we can find a domain $D$, symmetric about and containing the interval $[0,1]$ on which $F$ is analytic. If there exists a polynomial $G$ mapping $D$ conformally onto a domain containing the unit disc; and if $G$ is real on the reals with $G(0)=0$ and $G(1)=1$; then $F\left[G^{-1}(w)\right]$ is analytic on the closed unit disc; real on the reals and positive on $[0,1]$. Thus, by Lemma $17, F\left[G^{-1}(\cdot)\right] \in H$ and by Lemma 18 $F\left[G^{-1}(G(\cdot))\right]=F(\cdot) \in H$. Therefore, to complete the proof we need only show that $G$ exists.

Let $G_{1}(z)$ be the unique conformal mapping of $D$ onto the disc $|z|<1$, where $G_{1}(0)=0$ and $G_{1}^{\prime}(0)>0$. Since $D$ is symmetric about the interval $[0,1],\left[G_{1}\left(z^{*}\right)\right]^{*}$ also maps $D$ conformally onto $|z|<1$ and hence $\left[G_{1}\left(z^{*}\right)\right]^{*}=G_{1}(z)$ and $G_{1}$ is real on the real axis. Let $G_{2}(z)=\left[G_{1}(1)\right]^{-1} G_{1}(z)$. Then $G_{2}$ maps $D$ conformally onto the disc $|z|<\left[G_{1}(1)\right]^{-1}$. Clearly $G_{2}$ is real on the real axis with $G_{2}(0)=0$ and $G_{2}(1)=1$. Let $G_{3}(z)$ be a polynomial for which $G_{3}(0)=0$ and $\left|G_{2}(z)-G_{3}(z)\right|<\varepsilon$ for all $z \in D$, and define $G(z)$ as $a\left[G_{3}(z)+G_{3}\left(z^{*}\right)^{*}\right]$ where $a=\left[G_{3}(1)+G_{3}(1)^{*}\right]^{-1}$. Clearly we have $(2+2 \varepsilon)^{-1} \leqq|a| \leqq(2-2 \varepsilon)^{-1}$. If $D^{\prime}$ is the inverse image of the disc

$$
|w|<(2-2 \varepsilon)^{-1}\left[1+\left[G_{1}(1)\right]^{-1}-2 \varepsilon\right]-\varepsilon(1-\varepsilon)^{-1}
$$

under the mapping $G$, and $C$ is the inverse image of the circle $|w|=2^{-1}\left[1+\left[G_{1}(1)\right]^{-1}\right]$ under the mapping $G_{2}$; then $G_{2} \neq 0, \infty$ on $C$ and

$$
2 a G_{2}(z)-w=G(z)-w+h(z)
$$


where $|G(z)-w|>\varepsilon(1-\varepsilon)^{-1}>|h(z)|$ for all $z \in C$ and $w \in G\left(D^{\prime}\right)$. Thus, by Rouché's theorem, $G(z)$ takes on the value $w$ only once. Therefore, since $G\left(D^{\prime}\right)$ is a disc containing the unit disc for $\varepsilon$ sufficiently small and since $G$ is a 1-1 mapping of $D^{\prime}$ onto the disc $G\left(D^{\prime}\right)$ with $G(0)=0, G(1)=1$ and $G$ real on the real axis; the proof is complete.

\section{BIBLIOGRAPHY}

1. F. Hausdorff, Momentproblem für ein endliches Interval, Math. Z. 16 (1923), 220-248.

2. M. Kac, Probability and related topics in physical sciences, Interscience, New York, 1959.

3. H. P. McKean, Jr., An exponential formula for solving Boltzmann's equation for a Maxwellian gas, J. Combinatorial Theory 2 (1967), 358-382.

UNIVERSITY OF CALIFORNIA,

Riverside, CALIFornia 